\title{
Quantum mechanics basis of quality control in hard metals
}

\author{
Ruiwen Xie ${ }^{\mathrm{a}, *}$, Raquel Lizárraga ${ }^{\mathrm{a}, * *}$, David Linder ${ }^{\mathrm{a}}$, Ziyong $\mathrm{Hou}^{\mathrm{a}}$, Valter Strömª ${ }^{\mathrm{a}}$, Martina Lattemann ${ }^{\mathrm{b}}$, Erik \\ Holmström ${ }^{\mathrm{b}}$, Wei Li ${ }^{\mathrm{a}}$, Levente $\mathrm{Vitos}^{\mathrm{a}, \mathrm{c}, \mathrm{d}}$

\begin{abstract}
${ }^{a}$ Applied Materials Physics, Department of Materials Science and Engineering, Royal Institute of Technology, Stockholm SE-10044, Sweden ${ }^{b}$ Sandvik Coromant RED, Stockholm SE-12680, Sweden.

${ }^{c}$ Department of Physics and Astronomy, Division of Materials Theory, Uppsala University, Uppsala, Sweden

${ }^{d}$ Wigner Research Centre for Physics, Institute for Solid State Physics and Optics, Budapest, Hungary
\end{abstract}

\begin{abstract}
Non-destructive and reliable quality control methods are a key aspect to designing, developing and manufacturing new materials for industrial applications and new technologies. The measurement of the magnetic saturation is one of such methods and it is conventionally employed in the cemented carbides industry. We present a general quantum mechanics based relation between the magnetic saturation and the components of the binder phase of cemented carbides, which 'can be directly employed as a quality control. To illustrate our results, we calculate the magnetic saturation of a binder phase, $85 \mathrm{Ni} 15 \mathrm{Fe}$ binary alloy, using ab-initio methods and compare the theoretical predictions to the magnetic saturation measurements. We also analyse interface and segregation effects on the magnetic saturation by studying the electronic structure of the binder phase. The excellent agreement between calculations and measurements demonstrates the applicability of our method to any binder phase. Since the magnetic saturation is employed to ensure the quality of cemented carbides, the present method allows us to explore new materials for alternative binder phases efficiently.
\end{abstract}

Keywords: Hard metal, binder phase, magnetic saturation, ab-initio calculations

\section{Introduction}

Cemented carbides, also known as hard metals, are relatively tough and fatigue resistant materials used in cutting tools and rock drilling inserts, among other industrial applications. Essentially, hard metals are composite materials made of tungsten carbide (WC) grains glued together by a binder phase. Typically, the binder phase consists of ductile cobalt and some amount of dissolved tungsten and carbon. The microstructure of these materials is predominantly controlled by the components of the binder phase. For example, the grain growth rate is slower in W-rich binders, while faster and more homogeneous in Crich binders [1]. Moreover, the morphology and polymorphism of the WC-Co alloys are shown to be significantly influenced by the dissolved amount of $\mathrm{W}$ in the binder phase [2, 3]. Therefore, the knowledge of the composition of the binder phase, can be used to predict microstructural properties of hard metals.

Despite the great success of Co as a binder phase, the need of finding a substitute has been recognized because of cobalt's rising price and health threats [4, 5]. One important criterion for an appropriate substitute is that the range of the total $\mathrm{C}$ concentration in the sample, the socalled carbon window, should be relatively broad for the

\footnotetext{
* Corresponding author

${ }^{* *}$ Corresponding author

Email addresses: ruiwen2@kth.se (Ruiwen Xie), raqli@kth.se (Raquel Lizárraga )
}

sake of industrial manufacturing processes, so that the undesired $\eta$-phase $\left(\mathrm{M}_{x} \mathrm{C}\right)$ and graphite can be avoided easily. A series of $\mathrm{WC}-(\mathrm{Fe}, \mathrm{Co}, \mathrm{Ni})$ cemented carbides, which possesses comparable properties to Co-bonded hard metals, such as hardness, wear resistance and strength, was developed by Prakash et al. [ 6, 7]. In addition, the Fe-Ni-W$\mathrm{C}$ phase diagram calculated by Guillermet demonstrated that the carbon window widens with the increase of $\mathrm{Ni}$ concentration 8]. The WC-(Fe, Mn) hard metals were also reported to be promising alternative binders due to their higher hardness and comparative fracture toughness [9]. Recently, a cutting insert made of a new cemented carbide with a high entropy alloy as a binder phase showed superior performance in a machining test compared to a state-of-the-art cutting insert made of a Co-bonded hard metal [10, 11].

The measurement of the magnetic saturation is commonly used to identify the composition of the binder phase and to determine the localization of the sample in the carbon window, which is an important indicator of the properties of the composite [12, 13]. By measuring the change of the magnetic saturation, the $\mathrm{W}$ concentration dissolved into the binder phase and the overall $\mathrm{C}$ content in the composite can be non-destructively estimated [14]. Therefore, this measurement is routinely employed in the cutting tool industry as a quality control for cemented carbides. Such a quantitative relationship between the magnetic saturation and $\mathrm{W}$ composition in the binder has been constructed 
for WC-Co alloys 13, 15]. Unfortunately, today there is no such relation for a more general binder consisting of an alloy, which severely hampers the development of more complex binder phases. Hence, to obtain such a relationship is critical for the production of new Co-free cemented carbides.

In the present study, we formulate and compute a quantitative relationship between the magnetic saturation and the components of the binder phase using ab-initio methods. As an example, we use a binary $85 \mathrm{Ni} 15 \mathrm{Fe}$ alloy as a binder to illustrate this relationship, however, our modelling method is general and can be applied to more complex multi-component alloys. The theoretical results are then compared to experimental measurements of the weight specific magnetic saturation to demonstrate the applicability of our model. We also investigate the effects of metal/ceramic interfaces and segregation of the binder elements to the dissolved components in the binder phase on the magnetic saturation.

The paper is organized as follows: in Sec. 2 we derive the mathematical model to calculate the weight specific magnetic saturation and in Sec. 2.1, we introduce the computational model. Ab-initio methods are described in Sec. 2.2. Results and discussions are shown in Sec. 3 and finally in Sec. 4 we summarize our findings.

\section{Theory}

The carbon level of cemented carbides can be determined in a non-destructive way by measuring the weight specific magnetic saturation, $\sigma_{s}$, as discussed in Ref. [14]. Early work by Roebuck and Almond on Co-W-C alloys, demonstrated that the magnetic saturation decreases linearly with respect to $\mathrm{W}$ content [15]. Here we derive an expression to calculate the weight specific magnetic saturation for cemented carbides with a generic binder and we calculate it using ab-initio methods.

The weight specific magnetic saturation can be expressed as

$$
\sigma_{s}=\frac{\sum_{i} \mu_{i}}{\sum_{i} m_{i}},
$$

where $\mu_{i}$ and $m_{i}$ are the atomic magnetic moments and the masses of all the elements in the material, respectively. In particular, a hard metal only contains magnetic specimens in the binder, and if we consider an ideal binder, in which neither $\mathrm{C}$ - nor $\mathrm{W}$-diffusion occurs into the binder phase, the magnetic saturation can be written as

$$
\sigma_{s}^{b}=\frac{\sum_{j}^{N^{b}} \mu_{j}^{b}}{\sum_{i} m_{i}}=\frac{N^{b} \mu^{b}}{\sum_{i} m_{i}} .
$$

The index $j=\left\{1, N^{b}\right\}$, where $N^{b}$ is the number of atoms in an ideal binder, the quantity $\mu_{j}^{b}$ is the atomic magnetic moment of a binder atom without diffusion of $\mathrm{W}(\mathrm{C})$ and $\mu^{b}$ is the average magnetic moment of the binder atoms. However, both $\mathrm{W}$ and $\mathrm{C}$ do dissolve into the binder during sintering, causing the magnetic moments of their neighbouring binder atoms to change. The effect of the dissolved $\mathrm{W}$ and $\mathrm{C}$ on the magnetic saturation is then described by the ratio,

$$
\begin{aligned}
\frac{\sigma_{s}}{\sigma_{s}^{b}}=\frac{\sum_{i} \mu_{i}}{\sum_{j} \mu_{j}^{b}} & =\frac{N^{b} \mu^{b}+N_{W} \mu_{W}^{\mathrm{eff}}+N_{c} \mu_{C}^{\mathrm{eff}}}{N^{b} \mu^{b}} \\
& =1+\frac{N_{W} \mu_{W}^{\mathrm{eff}}}{N^{b} \mu^{b}}+\frac{N_{c} \mu_{C}^{\mathrm{eff}}}{N^{b} \mu^{b}} .
\end{aligned}
$$

The quantities $N_{W}$ and $N_{C}$ are the number of $\mathrm{W}$ and $\mathrm{C}$ atoms dissolved in the binder, respectively. The variables $\mu_{W}^{\mathrm{eff}}$ and $\mu_{C}^{\mathrm{eff}}$ correspond to the effective magnetic moment changes induced by $\mathrm{W}$ and $\mathrm{C}$, respectively and we define them as

$$
\mu_{W}^{\mathrm{eff}}=\mu_{W}+\delta \mu_{W}
$$

and

$$
\mu_{C}^{\mathrm{eff}}=\mu_{C}+\delta \mu_{C},
$$

where $\delta \mu_{W}$ and $\delta \mu_{C}$ denote the $\mathrm{W}$-induced and C-induced magnetic moment changes of the binder host, respectively. $\mu_{W}$ and $\mu_{C}$ are the magnetic moments of $\mathrm{W}$ and $\mathrm{C}$ in the binder phase, respectively. One can re-write Eq. 3 using the atomic concentration of $\mathrm{W}$ and $\mathrm{C}\left(C_{W}\right.$ and $\left.C_{C}\right)$ in the binder to obtain the following expression

$$
\begin{aligned}
\frac{\sigma_{s}}{\sigma_{s}^{b}}=1+( & \left.\frac{C_{W}}{1-C_{W}-C_{C}}\right) \frac{\mu_{W}^{\mathrm{eff}}}{\mu^{b}} \\
& +\left(\frac{C_{C}}{1-C_{W}-C_{C}}\right) \frac{\mu_{C}^{\mathrm{eff}}}{\mu^{b}}
\end{aligned}
$$

By ignoring the contribution of $\mathrm{C}$ to the weight specific magnetic saturation, $C_{C}=0$ in Eq. [5] and assuming a small concentration of $\mathrm{W}$ in the binder, one recovers the linear phenomenological expression derived by Roebuck and Almond for Co binders [15, 16].

In the following section we present the computational models to simulate the binder phase and calculate $\mu_{W}^{\text {eff }}$ and $\mu_{C}^{\mathrm{eff}}$ from first principles.

\subsection{Computational model}

In order to calculate $\mu_{W}^{\text {eff }}$ and $\mu_{C}^{\text {eff }}$ in Eqs. 4a and 4b we use the computational models shown in Fig. 19) and b), respectively. In our calculations we adopt the face-centred cubic (fcc) structure as a model, however our method is applicable to any crystal structure. Figure [1]) displays a $3 \times 3 \times 3$ fcc supercell for a binder, $85 \mathrm{Ni} 15 \mathrm{Fe}$, in which one binder atom has been substituted by a $\mathrm{W}$ atom, while Fig. 1b) corresponds to a supercell where one $\mathrm{C}$ atom occupies the octahedral interstitial position, which is verified to be the most stable occupation. In the following we assume that the dissolved $\mathrm{W}$ and $\mathrm{C}$ atoms do not interact with each other in the binder phase. This assumption is 


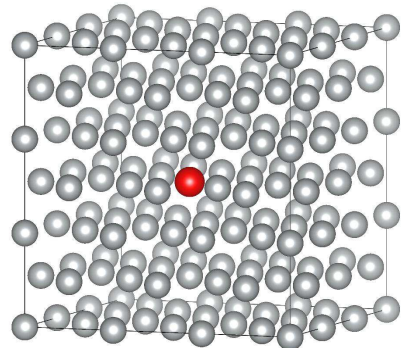

(a)

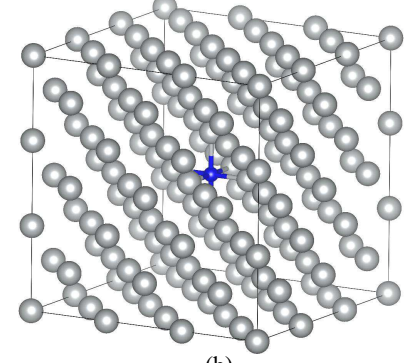

(b)
Figure 1: (Colour online) The computational models (a) Wsubstitutional binder phase and (b) C-interstitial binder phase, in which the red and blue spheres correspond to $\mathrm{W}$ and $\mathrm{C}$ atoms, respectively. The grey spheres are binder atoms. The number of binder atoms, $N_{b}$, is 107 in a) and 108 in b).

justified because according to observations $\mathrm{W}$ and, particularly, $\mathrm{C}$ concentrations $(<0.9$ at. \%) are quite small in the binder phase [17]. Therefore, the interaction between dissolved $\mathrm{W}$ and $\mathrm{C}$ in the binder phase can be neglected. We calculate $\delta \mu_{W(C)}$ then as the difference in total magnetic moment between an ideal binder and a binder in which $\mathrm{W}(\mathrm{C})$ diffusion has occurred

$$
\delta \mu_{W}=\sum_{i}^{N_{s}^{b}} \mu_{i}-\sum_{i}^{N_{s}^{b}} \mu_{i}^{b}
$$

and

$$
\delta \mu_{C}=\sum_{i}^{N_{s}^{b}} \mu_{i}-\sum_{i}^{N_{s}^{b}} \mu_{i}^{b}
$$

Here, $N_{s}^{b}$ denotes the number of binder atoms in the supercells shown in Fig. 1. namely $N_{s}^{b}=107$ in Fig. 11a) and $N_{s}^{b}=108$ in Fig. 10). The magnetic moments of binder atoms with $\left(\mu_{i}\right)$ and without $\mathrm{W}$ and $\mathrm{C}$ diffusion $\left(\mu_{i}^{b}\right)$ can be obtained from ab-initio calculations carried out for the supercells in Fig. 1,

\subsection{Ab-initio Method}

Most of the calculations are performed using a method based on density functional theory (DFT) [18, 19], the exact muffin-tin orbitals (EMTO) method [20, 21]. In the present work, we choose the binder $85 \mathrm{Ni} 15 \mathrm{Fe}$ as an example since it is a promising candidate for alternative binders in cemented carbides. In order to simulate the binder alloy $85 \mathrm{Ni} 15 \mathrm{Fe}$, we use the coherent potential approximation (CPA) 20, 22]. CPA is a well-known approach that allows to treat random alloys within the single-site approximation [21]. In the self-consistent calculations, the oneelectron equations are solved within the scalar-relativistic approximation and the soft-core scheme. $s, p, d, f$ orbitals are included in the muffin-tin basis set. The generalizedgradient approximation (GGA) of Perdew-Burke-Ernzerhof (PBE) 23] is employed as the exchange-correlation functional. The potential sphere radius of interstitial carbon
Table 1: Ab-initio calculated values of the magnetic moments of $\mathrm{W}$, the $\mathrm{W}$-induced magnetic moment change in the binder host, the magnetic moment of $\mathrm{C}$, the $\mathrm{C}$-induced magnetic moment change in the binder host and the average magnetic moment of the binder atoms.

\begin{tabular}{cccccc}
\hline & $\mu_{W}$ & $\delta \mu_{W}$ & $\mu_{C}$ & $\delta \mu_{C}$ & $\mu^{b}$ \\
& $\left(\mu_{B}\right)$ & $\left(\mu_{B}\right)$ & $\left(\mu_{B}\right)$ & $\left(\mu_{B}\right)$ & $\left(\mu_{B}\right)$ \\
\hline $85 \mathrm{Ni15Fe}$ & -0.37 & -3.98 & -0.08 & -2.96 & 0.95 \\
\hline
\end{tabular}

is optimized to be $0.77 w_{\mathrm{C}}^{0}$, where $w_{\mathrm{C}}^{0}$ is the atomic sphere radius of the corresponding Voronoi polyhedron around the $\mathrm{C}$ site. The coordinates of the first nearest neighbours around $\mathrm{C}$ in the EMTO method are set according to the optimized configuration of C-interstitial pure Ni using Vienna Ab-initio Simulation Package (VASP) [24, 25]. The relaxation in the $\mathrm{W}$-substitutional supercell is not considered here since the $\mathrm{W}$ substitution only induces slight expansion of its first nearest neighbours (10\% of the Cinduced expansion). Cell size tests were performed and we found that the results were fully converged for $3 \times 3 \times 3$ supercells. K-point grids were also carefully tested to ensure convergence and a grid of $5 \times 5 \times 5$ was used in the calculations. In the present work, the segregation energy calculations are performed by EMTO and the interface calculations by VASP.

\section{Results and discussion}

In order to calculate the weight specific magnetization using Eqs. 6a and 6b we used the computational models in Fig. 17) and b). The optimized lattice parameters for Wsubstitutional and C-interstitial supercells are both $3.55 \AA$. Table1lists the calculated values for the magnetic moment of $\mathrm{W}\left(\mu_{W}\right)$, the $\mathrm{W}$-induced magnetic moment change in the binder host $\left(\delta \mu_{W}\right)$, the magnetic moment of $\mathrm{C}\left(\mu_{C}\right)$, the $\mathrm{C}$-induced magnetic moment change in the binder host $\left(\delta \mu_{C}\right)$ and the average magnetic moment of the binder atoms $\left(\mu^{b}\right)$. The calculated value for $\mu^{b}$ in the bulk is 0.95 $\mu_{B}$, which is in perfect agreement with the Slater-Pauling curve [26]. We then obtain the following expression by inserting these calculated values into Eq. [5]

$$
\begin{aligned}
\frac{\sigma_{s}}{\sigma_{s}^{b}}=1-4.58 & \left(\frac{C_{W}}{1-C_{W}-C_{C}}\right) \\
& -3.19\left(\frac{C_{C}}{1-C_{W}-C_{C}}\right) .
\end{aligned}
$$

We note here that the effect of $\mathrm{W}$ - and C-diffusion is to reduce the weight specific magnetic saturation. Moreover, the influence of $\mathrm{W}$ on the magnetic moment is only significant to the limit of its third-nearest neighbours.

The magnetic saturation of cemented carbides measured by instruments in industry is obtained in units of 


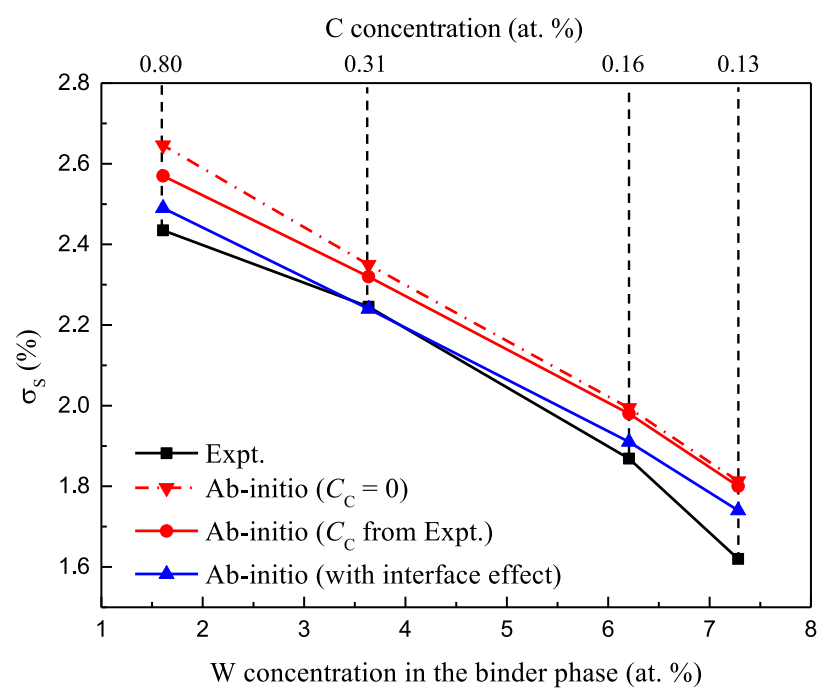

Figure 2: Weight specific magnetic saturation calculated using Eqs. 7 and 8 is shown by upside down triangles when $\mathrm{C}$ content is neglected and by circles when $\mathrm{C}$ concentration is taken from experimental estimations [17]. Triangles are used to indicate magnetic saturation including interface effects and the $\mathrm{C}$ content is taken from experiments. The experimental measurements [17] are denoted by squares.

the intrinsic Co weight specific magnetization [17]. Here, in order to compare directly with industrial measurements, we also express the magnetic saturation in the same unit, and thus we multiply Eq. 7 by the following factor

$$
F_{\mathrm{Co}}=\left(\frac{\mu^{b}}{\mu_{\mathrm{Co}}}\right) \frac{m_{\mathrm{Co}}}{m^{b}} C^{b} .
$$

In Eq. 8. $m_{\mathrm{Co}}$ is the atomic mass of Co, $\mu_{\mathrm{Co}}=1.67 \mu_{B}$ is the atomic magnetic moment of Co, $C^{b}=M^{b} / M^{\text {tot }}$ and $M^{b}$ and $M^{\text {tot }}$ are the masses of the binder and sample, respectively.

Eq. 7 contains two variables $C_{W}$ and $C_{C}$ that are known to be inversely related. However, the exact relationship may vary depending on many parameters, namely, grain size, diffusion rates, etc. To first approximation we ignore the carbon effect in Eq. 7 as it was done in Ref. [15] and we show the magnetic saturation as a function of $\mathrm{W}$ concentration by the dash-dotted line in Fig. 2. The magnetic saturation measurements have been performed on NiFe-WC using a vibrating sample magnetometer by Linder et al. [17, 27] and are depicted by squares in Fig. 2. They estimated $C_{W}$ and $C_{C}$ from a kinetic simulation method 27, 28]. We also employed their estimated $C_{W}$ and $C_{C}$ values to calculate the magnetic saturation and the results are presented by circles in Fig. 2

We first observe that the calculated weight specific magnetic saturation decreases with $\mathrm{W}$ concentration in the binder phase and that the agreement between theory and experiments is remarkably good. Moreover, by comparing the curves with and without $\mathrm{C}$ effect, it is clear that for small concentrations of $\mathrm{C}$ its influence on the magnetic sat- uration is negligible. Eq. 7 can then be used to predict the $\mathrm{W}$ concentration in the binder phase and hence it make it possible to predict the microstructural properties of hard metals.

During this analysis we have neglected the effects of segregation and interfaces. Thus, in the following sections, we will investigate the contribution of these two effects separately.

\subsection{Segregation effect on magnetic properties}

In order to estimate the effect of segregation in the binder alloy we will here develop a simple model that can be evaluated by means of our simulation method. The coherent potential approximation used in this investigation treats the binder alloy, $85 \mathrm{Ni} 15 \mathrm{Fe}$, as an ideal effective medium. However, in practice, segregation of the binder elements to the dissolved $\mathrm{W}$ or $\mathrm{C}$ may be present. Such segregation effects could alter the calculated values for the magnetic saturation due to the different magnetic responses of $\mathrm{W}$ and $\mathrm{C}$ to their neighbours: $\mathrm{Ni}$ and $\mathrm{Fe}$ (see the upper part of Table 2). The segregation energy of the $85 \mathrm{Ni} 15 \mathrm{Fe}$ alloy is calculated following the work of Delczeg et al. 29] and using the supercells in Fig. 1,

$$
\begin{aligned}
E_{\mathrm{segr}}=\left.\frac{d E_{1 \mathrm{nn}}\left(\mathrm{Ni}_{0.85+x} \mathrm{Fe}_{0.15-x}\right)}{d x}\right|_{x=0} & \\
& -\left.\frac{d E_{\mathrm{Bulk}}\left(\mathrm{Ni}_{0.85+x} \mathrm{Fe}_{0.15-x}\right)}{d x}\right|_{x=0},
\end{aligned}
$$

where $x$ is in this case the atomic concentration change for $\mathrm{Ni}$ and $\mathrm{Fe}$ and it is chosen to be 0.001. The two derivatives in Eq. 9 are calculated at constant volume. The total energy of the supercell is denoted by $E_{1 n n}\left(\mathrm{Ni}_{0.85+x} \mathrm{Fe}_{0.15-x}\right)$ if only the first nearest neighbours of the impurity $\mathrm{W}(\mathrm{C})$ are replaced by $\mathrm{Ni}_{0.85+x} \mathrm{Fe}_{0.15-x}$, whereas if instead all sites in the supercell are modified, the total energy corresponds then to $E_{\text {Bulk }}\left(\mathrm{Ni}_{0.85+x} \mathrm{Fe}_{0.15-x}\right)$. According to this definition, a positive $E_{\text {segr }}$ indicates that Fe prefers to be neighbour to $\mathrm{W}(\mathrm{C})$ instead of $\mathrm{Ni}$.

The segregation energy is calculated for the ferromagnetic state and it is $11.1 \mathrm{mRy}$ in the case of substitutional $\mathrm{W}$ and $12.4 \mathrm{mRy}$ in the case of interstitial C. This indicates that $\mathrm{Ni}$ tends to stay far away from the dissolved $\mathrm{W}$ and $\mathrm{C}$ atoms. However, one should keep in mind that the segregation unavoidably lowers the entropy of the binder phase. Therefore, we also calculate the entropy of a totally disordered $85 \mathrm{Ni15Fe}-\mathrm{W}(\mathrm{C})$ alloy,

$$
S=n_{1 n} c_{\mathrm{Ni}} \ln c_{\mathrm{Ni}}+n_{1 n} c_{\mathrm{Fe}} \ln c_{\mathrm{Fe}},
$$

where $n_{1 n}$ is the number of the first nearest neighbours of $\mathrm{W}(\mathrm{C}) \cdot n_{1 n}$ is equal to 12 and 6 in $\mathrm{W}$-substitutional and C-interstitial systems, respectively. $c_{\mathrm{Ni}}$ and $c_{\mathrm{Fe}}$ are the atomic concentrations of $\mathrm{Ni}$ and $\mathrm{Fe}$, respectively. The entropy is calculated to be $-3.21 \times 10^{-2} \mathrm{mRy} / \mathrm{K}$ and $-1.61 \times 10^{-2}$ $\mathrm{mRy} / \mathrm{K}$ for $\mathrm{W}$-substitutional and C-interstitial $85 \mathrm{Ni} 15 \mathrm{Fe}$ 
alloys, respectively. The temperatures, above which the entropy of the binder phase dominates the total energy, are then $346 \mathrm{~K}$ and $770 \mathrm{~K}$, respectively. This means that the entropy contribution is dominating at both, the solidification temperature and the temperature where solid state diffusion stops in the case of W. Some segregation could still be expected in the $\mathrm{C}$ case but since the amount of dissolved $\mathrm{C}$ is very low in the binder phase, we conclude that segregation does not contribute significantly to the magnetic saturation.

\subsection{Interface effect on magnetic properties}

The weight fraction of the binder phase is relatively low ( $\sim 5$ wt. \%), which means that many atoms from the binder phase in fact sit close to the hard phase and thus might possess different magnetic state as compared to the bulk binder atoms. Here we address this question and estimate the binder/WC interface effect on the magnetic saturation. The magnetic behaviour of the binder elements near the interface is studied by constructing an interface model $\mathrm{Ni}(111) / \mathrm{WC}(0001)$. This interface model consists of the low-index $\mathrm{Ni}(111)$ surface in fcc $\mathrm{Ni}$ and $\mathrm{WC}(0001)$ surface in hexagonal close packed (hcp) WC as shown in Fig. 3 a). Here, the Fe/WC interface is not considered since $\mathrm{Ni}$ is the dominant component in the binder $85 \mathrm{Ni} 15 \mathrm{Fe}$.

Two Ni(111)/WC(0001) interface models with $\mathrm{W}$ - and C-terminated $\mathrm{WC}(0001)$ surfaces are considered and are shown in Fig. 31(a) and (b), respectively. The Ni/WC interfaces are modelled using the coherent interface approximation [30], in which the averaged lattice parameter from both surfaces is used for the interface models. Accordingly, $\mathrm{a}=2.70 \AA$ As adopted as the new lattice parameter of the $\mathrm{Ni} / \mathrm{WC}$ interface, while the individual c/a ratios of $\mathrm{Ni}(111)$ and $\mathrm{WC}(0001)$ surfaces are fixed. The mismatch between $\mathrm{Ni}(111)$ and $\mathrm{WC}(0001)$ surfaces is approximately $16.8 \%$. Although, this mismatch is large we expect that the magnetic moments of bulk $\mathrm{Ni}$ are not severely affected as it can be seen in Table $2\left(\sim 0.62 \mu_{B}\right.$ in the equilibrium bulk calculation and $\sim 0.70 \mu_{B}$ in the interface calculation). The interface geometry follows the stacking sequence of $\mathrm{Ni}(111)$ surface. The atoms near the interface are relaxed.

In order to investigate the interface stability we calculate the interface energy $\gamma_{\mathrm{Ni} / \mathrm{WC}}$ following the work in Ref. [31]. The interface energy is then calculated as

$$
\gamma_{\mathrm{Ni} / \mathrm{WC}}\left(\tilde{\mu}_{C}\right)=\gamma_{\mathrm{Ni}}+\gamma_{\mathrm{WC}}\left(\tilde{\mu}_{C}\right)-W_{\mathrm{sep}}
$$

where $W_{\text {sep }}$ is the ideal work of separation of the interface, $\gamma_{\mathrm{Ni}}$ and $\gamma_{\mathrm{WC}}\left(\tilde{\mu}_{\mathrm{C}}\right)$ are free surface energies of $\mathrm{Ni}(111)$ and $\mathrm{WC}(0001)$, respectively. The free surface energy of $\mathrm{WC}(0001)$ is a function of the chemical potential of carbon, $\tilde{\mu}_{\mathrm{C}}$, according to Siegel's work [32]. Since the C composition in the binder phase is unknown, the exact chemical potential of $\mathrm{C}$ is also unknown before hand, however we know that the $\mathrm{C}$ content should be between the $\eta$ and graphite phase. Subsequently, the chemical potential of $\mathrm{C}$ in graphite is taken as reference and $\tilde{\mu}_{C}-\tilde{\mu}_{C}$ (bulk) is
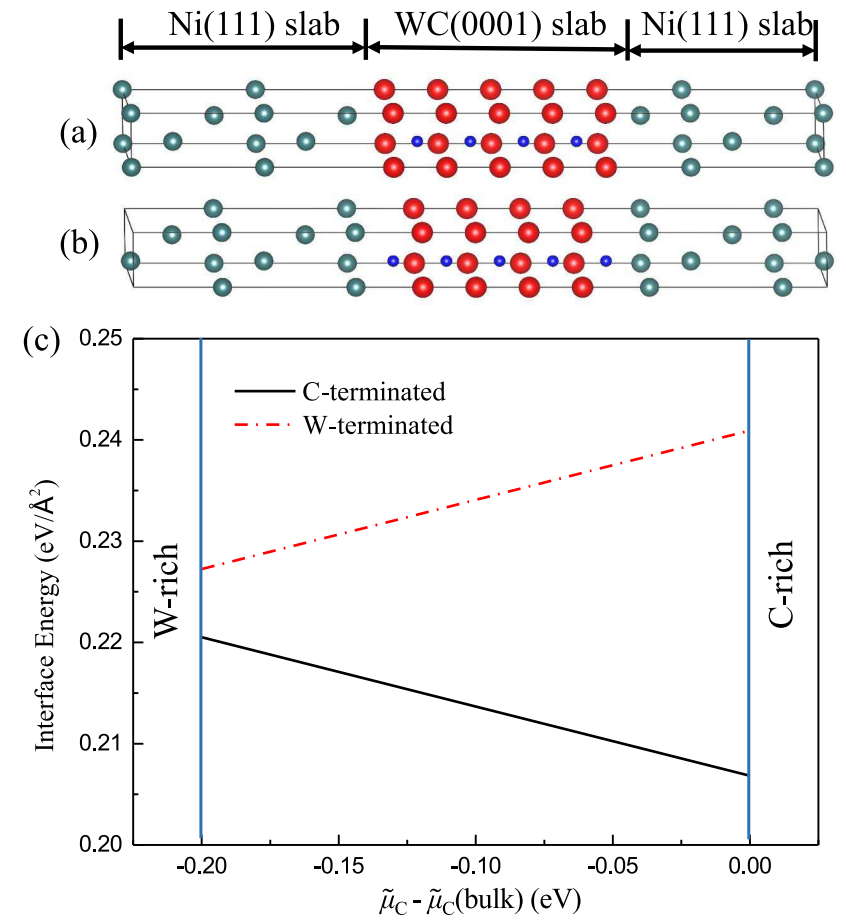

Figure 3: (a) W-terminated and (b) C-terminated $\mathrm{Ni}(111) / \mathrm{WC}(0001)$ interfaces. The red, blue and cyan balls represent $\mathrm{W}, \mathrm{C}$ and $\mathrm{Ni}$ atoms, respectively. (c) the calculated interface energy of W-terminated and C-terminated $\mathrm{Ni}(111) / \mathrm{WC}(0001)$ interfaces as a function of the chemical potential of $\mathrm{C}, \tilde{\mu}_{\mathrm{C}}$.

taken to be $\sim 0.2 \mathrm{eV}$ as in the WC-Co alloys [33]. The work of separation is defined as

$$
W_{\text {sep }}=\frac{1}{2 A}\left(E_{\mathrm{Ni}}+E_{\mathrm{WC}}-E_{\mathrm{int}}\right),
$$

where $E_{\mathrm{Ni}}$ and $E_{\mathrm{WC}}$ are the total energies of $\mathrm{Ni}$ and $\mathrm{WC}$ slabs, respectively, $E_{\text {int }}$ is the the total energy of the interface $\mathrm{Ni}(111) / \mathrm{WC}(0001) / \mathrm{Ni}(111)$ and $\mathrm{A}$ is the area of the interface. The free surface energies of $\mathrm{Ni}(111)$ and $\mathrm{WC}(0001)$ in Eq. 11 are computed using the following expressions

$$
\begin{aligned}
& \gamma_{\mathrm{Ni}}=\frac{1}{2 A}\left(E_{\mathrm{Ni}}^{s}-N_{\mathrm{Ni}} \tilde{\mu}_{\mathrm{Ni}}\right) \quad \text { and } \\
& \gamma_{W C}\left(\tilde{\mu}_{C}\right)=\frac{1}{2 A}\left(E_{\mathrm{WC}}^{s}-N_{W} \tilde{\mu}_{\mathrm{WC}}\right) \\
& \quad+\frac{1}{2 A}\left(N_{W}-N_{C}\right) \tilde{\mu}_{C},
\end{aligned}
$$

where $N_{\mathrm{Ni}}$ is the number of $\mathrm{Ni}$ atoms in $\mathrm{Ni}(111)$ surface model, $N_{W}$ and $N_{C}$ are the numbers of $\mathrm{W}$ and $\mathrm{C}$ atoms in $\mathrm{WC}(0001)$ surface model, respectively. In Eq. $13 \tilde{\mu}_{\mathrm{Ni}}$ and $\tilde{\mu}_{\mathrm{WC}}$ are the chemical potentials of bulk $\mathrm{Ni}$ and the $\mathrm{WC}$ unit in bulk WC, respectively. $E_{\mathrm{Ni}}^{s}$ and $E_{\mathrm{WC}}^{s}$ correspond to the total energies of $\mathrm{Ni}(111)$ and $\mathrm{WC}(0001)$ surfaces, respectively.

The calculated interface energies for C-terminated and W-terminated Ni/WC interfaces are presented in Fig. 3 (c) 
Table 2: Magnetic moments of $\mathrm{Ni}, \mathrm{Fe}, \mathrm{W}, \mathrm{C}$ in the bulk fcc model (Fig. 1) and Ni, W, C in the interface model (Fig 3). Ni* and Fe* denote the $\mathrm{Ni}$ and $\mathrm{Fe}$ atoms far away from the impurity.

\begin{tabular}{llllllll}
\hline \multirow{2}{*}{ Model } & & \multicolumn{5}{l}{ Magnetic moment $\left(\mu_{B}\right)$} \\
\cline { 3 - 8 } Bulk & & Ni-1n & Fe-1n & Ni* & Fe & C & W \\
\hline \multirow{2}{*}{ Interface } & C-alloying & 0.28 & 2.17 & 0.63 & 2.78 & -0.08 & - \\
& W-alloying & 0.38 & 2.47 & 0.62 & 2.78 & - & -0.37 \\
\hline
\end{tabular}

as a function of chemical potential of carbon. One can observe that the C-terminated $\mathrm{Ni} / \mathrm{WC}$ interface is thermodynamically more stable than the $\mathrm{W}$-terminated interface over the whole range of carbon chemical potential considered in the present work.

The effects of $\mathrm{C}$ and $\mathrm{W}$ on the magnetic moments of $\mathrm{Ni}$ around the interface can be seen in the lower part of Table 2. Table 2 shows that both $\mathrm{C}$ and $\mathrm{W}$ decrease the magnetic moments of the $\mathrm{Ni}$ atoms located at the interface. The atomic magnetic moments of the interfacial Ni atoms are reduced to $0.03 \mu_{B}$ and $0.24 \mu_{B}$ for C-terminated and $\mathrm{W}$-terminated interface models, respectively, from the value $0.70 \mu_{B}$ when $\mathrm{Ni}$ is far away from the interface. The magnetic moments of $\mathrm{C}$ and $\mathrm{W}$ near the interface are $0 \mu_{B}$ and $0.02 \mu_{B}$, respectively.

To estimate the upper limit of the interface effect, here we assume that all the present interfaces in cemented carbides are C-terminated and that the magnetic moments of the binder elements near the interface are approximately zero. Moreover, a tetrahedral shape is assumed for the binder phase. The size of the binder lakes is set to be 0.1 $\mu \mathrm{m}$, which is in the same quantitative level as reported in Ref. [2]. Based on these assumptions, the ratio of the number of surface atoms to the number of bulk atoms in the binder lake $n_{s} / n_{b}$ is calculated by

$$
\frac{n_{s}}{n_{b}}=\left(\frac{\sqrt{3} a^{2}}{\pi r^{2}}\right) \frac{\frac{4 \pi r^{3}}{3}}{\frac{\sqrt{2} a^{3}}{12}}=\frac{\sqrt{2} a}{16 \sqrt{3} r},
$$

where $a$ is the length of edge in the tetrahedron and $r$ is taken for simplicity as the atomic radius of $\mathrm{Ni}$ since the atomic radii of $\mathrm{Fe}$ and $\mathrm{Ni}$ are quite similar.

The weight specific magnetic saturation can be now evaluated including the calculated interface effect and the new curve can be seen in Fig. 2 (with triangles). One can see that the consideration of interface effect lowers the magnetic saturation and hence, the theoretically-predicted magnetic saturation becomes even closer to the experimental measurements. Here, we also need to mention that by considering the random shape distribution of the binder lakes in cemented carbides, the assumed shape with a lower surface-area-to-volume ratio would give a smaller interface effect on the absolute magnetic saturation value.
Furthermore, the W-terminated interface might also exist in the cemented carbides although our results show that it is thermodynamically less stable than the C-terminated interface.

Interestingly, we notice from Table 2 that $\mathrm{W}$ and $\mathrm{C}$ show different magnetic behaviours. In the bulk computational model (Fig. 1), C decreases the magnetic moment of $\mathrm{Ni}(\mathrm{Fe})$ more dramatically than $\mathrm{W}$, while $\mathrm{W}$ itself gains more negative magnetic moment. Such a difference can be explained with their corresponding density of states (DOS) displayed in Fig. 4. The $d$ electrons of $\mathrm{W}$ contributes to its negative magnetic moment while the electronic structures of its surrounding $\mathrm{Ni}$ and $\mathrm{Fe}$ atoms (Ni-1n, Fe-1n) are nearly unchanged compared to the $\mathrm{Ni}(\mathrm{Fe})$ atoms far away from the impurity $\left(\mathrm{Ni}^{*}, \mathrm{Fe}^{*}\right)$. In contrast, the spin-up and spin-down DOS are almost identical for $\mathrm{C}$, indicating the approximately zero magnetic moment of $\mathrm{C}$. The electronic structure of $\mathrm{C}$-neighboured $\mathrm{Ni}$ and $\mathrm{Fe}$ atoms changes more due to the presence of $\mathrm{C}$ than that of those atoms far away from C. Similar magnetic features can also be observed in the interface model; the magnetic moment of the interfacial $\mathrm{Ni}$ atom $\left(0.03 \mu_{B}\right)$ in the C-terminated interface is much lower than the one in the $\mathrm{W}$-terminated interface $\left(0.24 \mu_{B}\right)$. The different behaviours of $\mathrm{W}$ and $\mathrm{C}$ have also been observed in $\mathrm{Co}-\mathrm{W}$ and Ni-P alloys 34]. C and $\mathrm{W}$ atoms in the $\mathrm{Ni} / \mathrm{WC}$ interface show approximately zero magnetic moment due to the strong covalent bond between $\mathrm{C}$ and $\mathrm{W}$ in $\mathrm{WC}(0001)$ slabs.

\section{Conclusions}

In the present work, we build a theory-based relationship between the weight specific magnetic saturation and the components of the binder phase for cemented carbides WC-85Ni15Fe. This relationship predicts the amount of $\mathrm{W}$ in the binder phase of cemented carbides, and it constitutes a non-destructive quality control. The calculated magnetic saturation is in excellent agreement with the experimentally measured values.

The segregation of $\mathrm{Ni}(\mathrm{Fe})$ to dissolved $\mathrm{W}$ or $\mathrm{C}$ and the $\mathrm{Ni}-\mathrm{Fe} / \mathrm{WC}$ interface are investigated as well. The segregation study indicates that $\mathrm{Ni}$ tends to be segregated from the dissolved $\mathrm{W}$ or $\mathrm{C}$ in the binder phase. However, for the $\mathrm{W}$ - and C-dissolved binder phase, the entropy would dominate the total energy above 346 and 770 

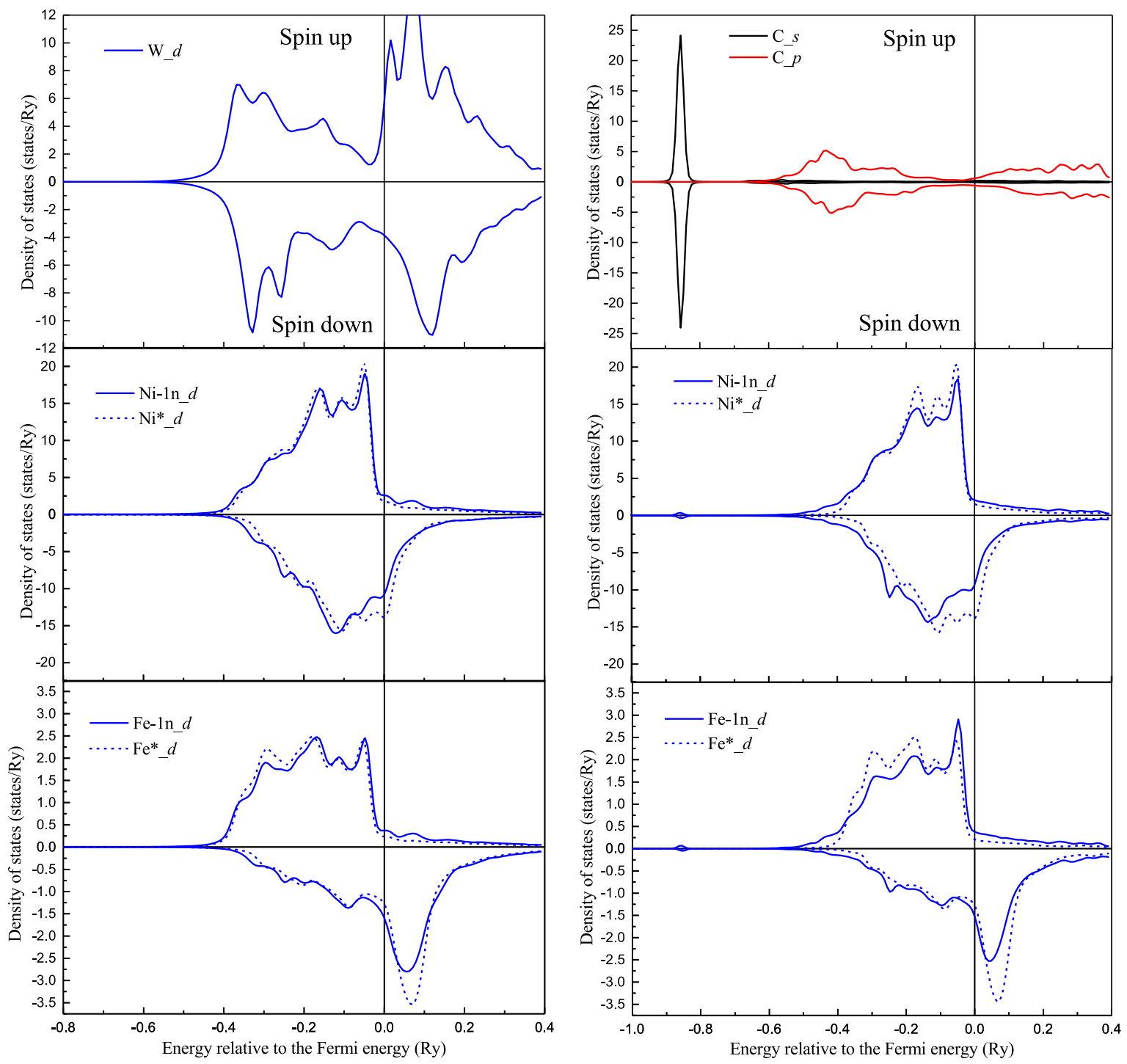

Figure 4: (a) Calculated density of states for W, Ni and Fe in the W-substitutional bulk fcc model (Fig. 1 a) and (b) calculated density of states for $\mathrm{C}, \mathrm{Ni}$ and $\mathrm{Fe}$ in the C-interstitial bulk fcc model (Fig. 1 b). Fe-1n and Fe* correspond to first nearest neighbor to the impurity and an atom far from the impurity, respectively.

K, respectively. The segregation effect on the magnetic saturation is therefore believed not to be significant. The $\mathrm{Ni}(111) / \mathrm{WC}(0001)$ interface is constructed and its stability is investigated from first principles. The interface effect on the magnetic saturation is estimated and by including this effect the magnetic saturation is reduced further, becoming even closer to experimental values.

Our results show that the magnetic behaviours of $\mathrm{W}$ and $\mathrm{C}$ are different in the binder phase. $\mathrm{W}$ decreases the total magnetic moment of the whole binder phase mainly by gaining itself negative magnetic moment, while $\mathrm{C}$ reduces the magnetic moments of its neighbours. Such distinct characteristics are explained by their corresponding electronic structure.

The present theoretical method provides a quantum mechanical approach to build a non-destructive quality control process for cemented carbides. Moreover, the comparison between theory and experiment helps to gain a physical insight into the magnetic properties of cemented carbides. This theoretical method can also be applied to other cemented carbides with multi-component binders and therefore significantly aiding the search for more complex binder phases for hard metals.

\section{Acknowledgments}

The present work is performed under the project COFREE (15048), funded by European Institute of Innovation \& Technology (EIT). The authors acknowledge the Ministry of Science and Technology (No.2014CB644001), the Swedish Research Council, the Swedish Foundation for Strategic Research, the Carl Tryggers Foundations, the Swedish Foun- 
dation for International Cooperation in Research and Higher Education, the Hungarian Scientific Research Fund (OTKA 128229), and the China Scholarship Council for financial supports. The computations were performed on resources provided by the Swedish National Infrastructure for Computing (SNIC) at Linköping.

\section{References}

\section{References}

[1] V. Chabretou, O. Lavergne, J.-M. Missiaen, C. Allibert, Quantitative evaluation of normal and abnormal grain growth of cemented carbides during liquid phase sintering, Met. Mater. 5 (2) (1999) 205-210.

[2] J. M. Marshall, M. Giraudel, The role of tungsten in the Co binder: Effects on WC grain size and hcp-fcc Co in the binder phase, Int. J. Refract. Met. Hard Mater. 49 (2015) 57-66.

[3] J. Weidow, H.-O. Andrén, Binder phase grain size in WC-Cobased cemented carbides, Scripta Mater. 63 (12) (2010) 11651168.

[4] H. Jobs, Powder metallurgy as a source of dust from the medical and technical standpoint, Vertravenargt 5 (1940) 142-148.

[5] A. Linna, P. Oksa, K. Groundstroem, M. Halkosaari, P. Palmroos, S. Huikko, J. Uitti, Exposure to cobalt in the production of cobalt and cobalt compounds and its effect on the heart, Occup. Environ. Med. 61 (11) (2004) 877-885.

[6] L. Prakash, et al., Properties of tungsten carbides with $\mathrm{Fe}-\mathrm{Co}-$ Ni alloys as binders, Sixth Int. Powder Metall. Conf., Dresden (1977) pp. 39-1.

[7] L. Prakash, Properties of tungsten carbides with an Fe-Co-Ni binder in sintered and heat-treated states, KFK-Nachr 11 (2) (1979) 35-42.

[8] A. F. Guillermet, An assessment of the $\mathrm{Fe}-\mathrm{Ni}-\mathrm{W}-\mathrm{C}$ phase diagram, Z. Metallkd. 78 (3) (1987) 165-171.

[9] C. Hanyaloglu, B. Aksakal, J. Bolton, Production and indentation analysis of $\mathrm{WC} / \mathrm{Fe}-\mathrm{Mn}$ as an alternative to cobalt-bonded hardmetals, Mater. Charac. 47 (3-4) (2001) 315-322.

[10] E. Holmström, R. Lizárraga, D. Linder, A. Salmasi, W. Wang, B. Kaplan, H. Mao, H. Larsson, L. Vitos, High entropy alloys: Substituting for cobalt in cutting edge technology, Appl. Mater. Today 12 (2018) 322-329.

[11] R. Lizárraga, E. Holmström, L. Vitos, Alloying effect of tungsten on the structural and magnetic properties of CoCrFeNiW high entropy alloys, Phys. Rev. Mat. 2 (2018) 094407.

[12] K. Brookes, Hardmetals and other hard materials international carbide data, East Barnet.

[13] A. Love, S. Luyckx, N. Sacks, Quantitative relationships between magnetic properties, microstructure and composition of WC-Co alloys, J. of Alloys and Compd. 489 (2) (2010) 465-468.

[14] A. Love, S. Luyckx, Hard materials: Characterisation: Quantitative relationships between magnetic properties, microstructure and composition of WC-Co, in: Eur. Congress and Exhibition on Powder Metallurgy. European PM Conference Proceedings, Vol. 1, The European Powder Metallurgy Association, 2006, p. 29.

[15] B. Roebuck, E. Almond, A. Cottenden, The influence of composition, phase transformation and varying the relative $\mathrm{FCC}$ and HCP phase contents on the properties of dilute Co-W-C alloys, Mater. Sci. Eng. 66 (2) (1984) 179-194.

[16] M. Lattermann, R. Xie, R. Lizárraga, L. Vitos, E. Holmström, Understanding quality control of hard metals in industry - a quantum mechanics approach. Manuscript in preparation.

[17] D. Linder, Z. Hou, R. Xie, P. Hedström, V. Ström, E. Holmström, A. Borgenstam, A comparative study of microstructure and magnetic properties of a Ni-Fe cemented carbide: Influence of carbon content. Manuscript submitted for consideration for publication.
[18] P. Hohenberg, W. Kohn, Inhomogeneous electron gas, Phys. Rev. 136 (1964) B864.

[19] W. Kohn, L. Sham, Self-consistent equations including exchange and correlation effects, Phys. Rev. 140 (1965) A1113.

[20] L. Vitos, I. Abrikosov, B. Johansson, Anisotropic lattice distortions in random alloys from first-principles theory, Phys. Rev. Lett. 87 (15) (2001) 156401.

[21] L. Vitos, Computational quantum mechanics for materials engineers: the EMTO method and applications, Springer Science \& Business Media, 2007.

[22] P. Soven, Coherent-potential model of substitutional disordered alloys, Phys. Rev. 156 (3) (1967) 809.

[23] J. P. Perdew, K. Burke, M. Ernzerhof, Generalized gradient approximation made simple, Phys. Rev. Lett. 77 (18) (1996) 3865 .

[24] G. Kresse, J. Hafner, Ab initio molecular dynamics for liquid metals, Phys. Rev. B 47 (1) (1993) 558.

[25] G. Kresse, D. Joubert, From ultrasoft pseudopotentials to the projector augmented-wave method, Phys. Rev. B 59 (3) (1999) 1758.

[26] J. Kübler, Theory of itinerant electron magnetism, Vol. 106, Oxford University Press, 2017.

[27] Z. Hou, D. Linder, P. Hedström, A. Borgenstam, E. Holmström, V. Ström, Effect of carbon content on the curie temperature of WC-NiFe cemented carbides, Int. J. Refract. Met. and Hard Mater. 78 (2019) 27-31.

[28] M. Walbrühl, D. Linder, J. Ågren, A. Borgenstam, Diffusion modeling in cemented carbides: Solubility assessment for Co, Fe and Ni binder systems, Int. J. Refract. Met. and Hard Mater. 68 (2017) 41-48.

[29] L. Delczeg, B. Johansson, L. Vitos, Ab initio description of monovacancies in paramagnetic austenitic $\mathrm{Fe}-\mathrm{Cr}-\mathrm{Ni}$ alloys, Phys. Rev. B 85 (17) (2012) 174101.

[30] Y. Li, Y. Gao, B. Xiao, T. Min, S. Ma, D. Yi, Theoretical calculations on the adhesion, stability, electronic structure, and bonding of Fe/WC interface, Appl. Surf. Sci. 257 (13) (2011) $5671-5678$.

[31] M. Christensen, G. Wahnström, Effects of cobalt intergranular segregation on interface energetics in WC-Co, Acta Mater. 52 (8) (2004) 2199-2207.

[32] D. J. Siegel, L. G. Hector Jr, J. B. Adams, Adhesion, stability, and bonding at metal/metal-carbide interfaces: Al/WC, Surf. Sci. 498 (3) (2002) 321-336.

[33] A. F. Guillermet, Thermodynamic properties of the Co-WC system, Metall. Trans. A 20 (5) (1989) 935-956.

[34] B. Szpunar, M. Aus, C. Cheung, U. Erb, G. Palumbo, J. A. Szpunar, Magnetism in nanostructured $\mathrm{Ni}-\mathrm{P}$ and $\mathrm{Co}-\mathrm{W}$ alloys, J. Mag. Mag. Mat. 187 (3) (1998) 325-336. 